\title{
DETONATION INITIATION TECHNIQUES FOR PULSE DETONATION PROPULSION
}

\section{S. M. Frolov}

The paper outlines recent efforts of the research team directed by the author on the development of efficient means for reducing the deflagrationto-detonation transition (DDT) run-up distance and time for liquidfueled air-breathing pulse detonation engine (PDE) applications. The main objective of the studies was to ensure fast DDT in a PDE tube at the lowest possible ignition energy, at the shortest distance, with the lowest pressure loss, and using aviation kerosene TS-1 (Russian analogue of JetA) as fuel and air as oxidizer.

\section{CONCEPT OF FAST DEFLAGRATION-TO-DETONATION TRANSITION}

Classical mechanism of DDT in a straight smooth-walled tube includes several stages [1, 2], namely: $(i)$ forced mixture ignition followed by formation of laminar flame; ( $i$ ) progressive increase of the burning velocity due to various instabilities and flow turbulization ahead of the propagating flame; $(i i i)$ formation and amplification of the shock wave ahead of the accelerating flame; $(i \nu)$ autoignition of the shock-compressed explosive mixture in the region between the shock wave and the flame [3] ("explosion in the explosion" [4]), leading to the formation of an overdriven detonation wave; and then $(\nu)$ self-sustaining Chapman-Jouguet (CJ) detonation. The detonation run-up distance and time are known to be determined mainly by stages $(i)$ to $(i i i)$ [5]. For the onset of detonation in hydrocarbon fuel-air mixtures, the apparent flame speed in the laboratory frame of reference should exceed $1000 \mathrm{~m} / \mathrm{s}$ [6]. At such a flame speed, the velocity of the shock wave propagating ahead exceeds $1300 \mathrm{~m} / \mathrm{s}$ (Mach number $\mathrm{M} \sim 3.8$ ), and pressure and temperature of the shock-compressed explosive gas exceed 1.7 MPa and $1200 \mathrm{~K}$, respectively.

A new approach of detonation initiation in a straight smooth-walled tube has been suggested recently [7-10]. It implies forced acceleration of a relatively weak shock wave traveling ahead of the flame up to the intensity sufficient for spontaneous detonation onset. For this purpose, a number of distributed ignition 
sources were mounted along the tube. To avoid poorly reproducible initial DDT stages $(i)$ to $($ iii $)$, the primary shock wave was generated either by an electric discharge [7-10] or by an additional tube section with Shchelkin spiral [10, 11].

In the experiments, the primary shock wave was amplified by triggering each successive ignition source coherently with the shock wave arrival to the corresponding tube cross section. By other words, the shock wave was amplified due to fast forced ignition of the explosive mixture in the close vicinity to the propagating shock front. This approach made it possible to initiate detonation at a distance and time considerably shorter as compared with the classical DDT. In the experiments [7-11] with detonation initiation, the time shift between shock wave arrival and gas ignition did not exceed $100 \mu \mathrm{s}$. At a larger time shift, detonation did not arise, other conditions being equal. Interestingly, the admissible value of the time shift correlates well with the characteristic reaction time in marginal detonation waves [12].

In the experiments [7-11] the detonation of the stoichiometric propane-air mixture was initiated in a tube $51 \mathrm{~mm}$ in diameter at the initial shock wave velocity at the level of $800-1000 \mathrm{~m} / \mathrm{s}$ (Mach number $\mathrm{M} \sim 2.4-3.0$ ). Note that to form such a shock wave ahead of the propagating flame front, the latter should accelerate to the apparent flame speed of about $550-750 \mathrm{~m} / \mathrm{s}$. This speed is considerably lower than the flame speed $(\sim 1000 \mathrm{~m} / \mathrm{s})$ required for spontaneous DDT. Therefore, if one makes a provision for the forced amplification of the shock wave ahead of the propagating flame, one can shorten the DDT run-up distance and time considerably, i.e., to ensure "fast" DDT.

From now on, the term "fast" DDT will be used implying the onset of detonation in the fuel-air mixture at the condition when the turbulent flame accelerates to the speed which is considerably lower than that required for the classical DDT in a straight tube. The classical DDT will be, therefore, referred to as the "slow" DDT [9] to distinguish it from fast DDT. The new approach for detonation initiation [7-10] was named as "detonation initiation by a traveling external ignition source" [9].

Turn over from the DDT in a straight smooth-walled tube to the DDT in a tube with regular obstacles $[2,13]$. The mechanism of DDT in such tubes is somewhat similar to the mechanism [1-6] described above. However, there exist some important differences. First, in the obstructed tube, the flame accelerates considerably faster due to additional turbulization of fresh explosive mixture by obstacles. Second, additional mechanisms of mixture ignition come into play: the mixture can autoignite due to reflections of the shock wave from obstacles or (in case of large obstacles) due to mixing of jets of hot combustion products with relatively cold explosive gas $[1,2]$.

A possibility of local autoignition of the reactive mixture at shock wave reflection from obstacles suggests an idea [9] that in tubes with obstacles, one can expect the existence of an alternative scenario of detonation initiation by a traveling spontaneous (rather than external) ignition source which supplements 
the classical DDT scenario [1-6]. In this case, mixture ignition in the vicinity of the shock wave propagating ahead of the flame occurs due to autoignition of the mixture behind a reflected shock wave rather than due to external stimulation of chemical activity.

By other words, in the course of DDT process in the obstructed tube, a particular stage seems to exist when fast detonation initiation by a traveling pulse of spontaneous ignition (fast DDT) is possible. Similar to the experiments on the DDT with external ignition sources [7-10], the feasibility of fast DDT is determined by the coherence of shock wave arrival to a particular tube cross section and mixture ignition in this section. In the case under consideration, one has to compare the shock wave arrival at an obstacle with the mixture autoignition behind the reflected shock wave. The latter is known to be characterized by the ignition delay time.

The ignition delay time depends on mixture composition as well as on shock wave intensity and compression phase duration. If one assumes that the admissible value of the ignition delay at normal shock reflection from an obstacle should be shorter than $100 \mu \mathrm{s}$, then for fast DDT in the stoichiometric propane-air mixture, one will need an incident shock wave traveling at the velocity of 950$970 \mathrm{M} / \mathrm{c}(\mathrm{M} \sim 2.8)[14]$. In the course of DDT, such a shock wave is generated by the flame propagating at a speed of about $700 \mathrm{~m} / \mathrm{s}$. At normal reflection of such a shock wave from an obstacle, the pressure and temperature attain the values of about $4.5 \mathrm{MPa}$ and $1300 \mathrm{~K}$. The estimated value of the shock wave velocity is well within the range of the shock velocities in the experiments on detonation initiation by a traveling external ignition source [7-10]. Hence, at the DDT stage when the flame speed in the stoichiometric propane-air mixture is well below $700 \mathrm{~m} / \mathrm{s}$, there are seemingly no prerequisites for fast DDT. At this stage, turbulent flame accelerates progressively and the shock wave is gradually amplified due to interaction with compression waves generated by the flame and by ignition spots arising in the vicinity to obstacles. When the flame speed exceeds the characteristic value of $700 \mathrm{~m} / \mathrm{s}$ and the shock wave velocity exceeds 950-970 m/s, fast DDT becomes theoretically possible.

Note that the estimates given above do not take into account various effects caused by expansion of the explosive mixture which inevitably come into play at shock wave diffraction at obstacles. These effects depend on the obstacle shape and result in increasing of the ignition delay time and, therefore, lead to the growth of the threshold flame speed required for fast DDT.

The possibility of 'fast' DDT raises fundamental questions regarding the conditions required for its realization. This paper provides the examples indicating feasibility of 'fast' DDT in the conditions when the flow behind a propagating shock wave experiences coherent localized ignitions. In the examples, only hydrocarbon fuels (propane, $n$-hexane, $n$-heptane, low Octane Number gasoline, and aviation kerosene) exhibiting relatively low detonability (detonation cell size is about $50 \mathrm{~mm}$ ) were considered. 


\section{SHAPED OBSTACLES}

In view of the possibility of fast DDT, the DDT run-up distance and time can be controlled by obstacle shape $[15,16]$. Surprisingly, the effect of obstacle shape on the propagating shock wave has not hitherto been studied, although the phenomenon of shock wave focusing in straight tubes after reflection from a nonflat end wall has long been known [17].

The computational and experimental studies described in this section demonstrate that 'fast' DDT can be facilitated by appropriate shaping of regular obstacles in the tube. Among different shapes of obstacles, orifice plates (Fig. 1a) and "saw-tooth" shaped obstacles (Fig. 1b) were chosen for consideration. The height and the total cross-section area of the orifice plates in the $x-y$ plane were kept similar to the shaped obstacles. The shape of "saw-tooth" obstacles was taken to be a combination of two parabolas $x_{1}=a_{1} y^{2}+b_{1} y+c_{1}\left(a_{1}>0\right)$ and $x_{2}=a_{2} y^{2}+b_{2} y+c_{2}\left(a_{2}<0\right)$ with the joint focus lying in the plane of symmetry of the channel at $y=H / 2$ and $x_{1 f}=x_{2 f}$. The points of intersection of the parabolas corresponded to the apices of obstacles of height $h$ located on the opposite walls of the channel. In the channel test section of length $L$, several identical obstacles were mounted at a distance $\delta=c_{1}-c_{2}$ from each other. Initially, the channel was filled with a quiescent, premixed, explosive gas at temperature $T_{0}$ and pressure $p_{0}$. A planar shock wave of initial Mach number M and compression phase duration $\tau$ was generated in the initiation section and, propagating from left to right, entered the obstructed section of the channel.

In the obstructed section, gas ignition caused by shock wave reflections from the obstacles and by interactions between various wave systems can occur. After

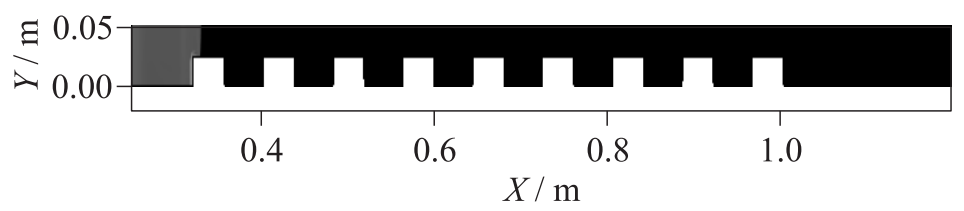

(a)

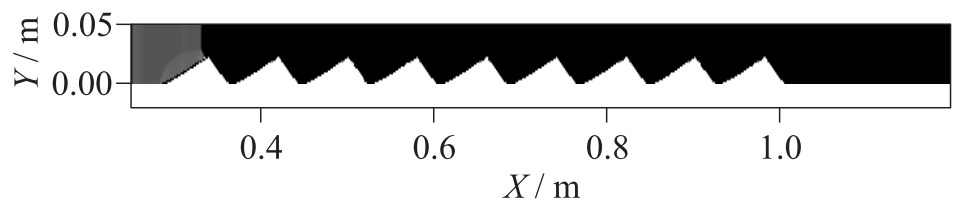

(b)

Figure 1 Shock wave enters the channel section with regular orifice plates $(a)$ and with regular shaped obstacles $(b)$. Only a lower part of the channel is shown in the figure 

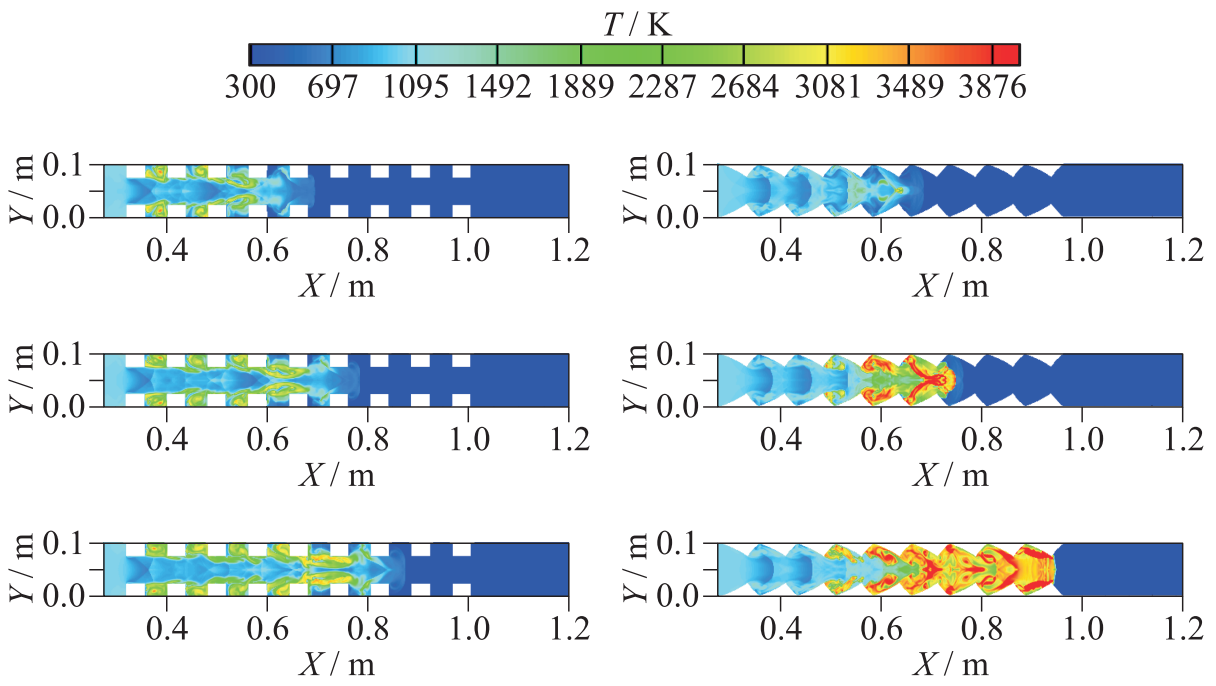

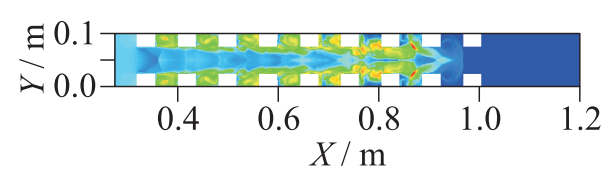

(a)

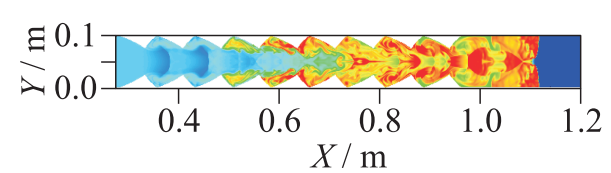

(b)

Figure 2 Snapshots of temperature fields in the channel with regular orifice plates $(a)$ and regular shaped obstacles $(b)$. The upper snapshot corresponds to $480 \mu \mathrm{s}$. Other snapshots are plotted with the time interval of $100 \mu \mathrm{s}$. (Refer Frolov, p. 325.)

ignition, two scenarios of the postshock flow evolution leading to detonation onset can be considered, namely, slow and fast DDT. This study is focused on the second, 'fast,' scenario. In this scenario, the stage of turbulent flame development and propagation is of minor importance as compared to fast energy deposition in the spontaneous ignition fronts propagating in the portions of the explosive gas preconditioned by multiple shock compression.

The mathematical model based on the full two-dimensional (2D) NavierStokes equations supplemented by the equation of energy conservation, equations of chemical kinetics, and the ideal gas equation of state was reported elsewhere [15]. The numerical procedure was based on the finite volume approach and Godunov flux approximation and was implemented for parallel computing. The explosive gas was the stoichiometric propane-air mixture at normal initial conditions.

Figure 2 shows the predicted snapshots of the temperature field in the channels with regular orifice plates (Fig. $2 a$ ) and regular shaped obstacles (Fig. 2b) 
with $c_{1}=0.008 \mathrm{~m}, c_{2}=0, h=0.025 \mathrm{~m}, x_{1 f}=x_{2 f}=0.065 \mathrm{~m}, H=0.1 \mathrm{~m}$, and $L=0.76 \mathrm{~m}$. The initial parameters of the shock wave were: $\mathrm{M}=3$ and $\tau \approx 800 \mu \mathrm{s}$. The calculations for the channel with orifice plates did not result in the detonation onset (Fig. 2a). Despite earlier ignition as compared to Fig. $2 b$, the orifice plates did not lead to fast DDT. Only with the initial shock of Mach number exceeding 4.5, it was possible to initiate detonation in the channel with orifice plates. In Fig. $2 b$, spontaneous ignition occurred in the flow core above the fifth obstacle in the vicinity to the focal point at about $490 \mu$ s (the upper snapshot). This ignition gave rise to a detonation. The detonation wave passed the obstructed section of the channel and propagated steadily in the smooth outlet section. When the initial shock wave entered the obstructed section from the right end, with other conditions being identical to those for Fig. $2 b$, no fast DDT was detected in the calculations. This test indicates the importance of the upwind and wake shapes of the obstacles. The computational results are discussed in detail elsewhere $[15,16]$.

For better understanding fast DDT phenomenon, the preignition spatial temperature distributions in the vicinity to the ignition site above the fifth obstacle in Fig. $2 b$ were analyzed. The analysis $[15,16]$ testifies that the detonation forms due to spontaneous generation of strong blast waves in the exothermic centers. The mechanism of blast wave formation due to propagation of fast spontaneous flames in the compressible medium was studied elsewhere [18].

For checking the findings described above, a series of experiments was performed. The experimental setup contained a high-pressure chamber (HPC) to generate shock waves of different initial intensity and a low-pressure chamber

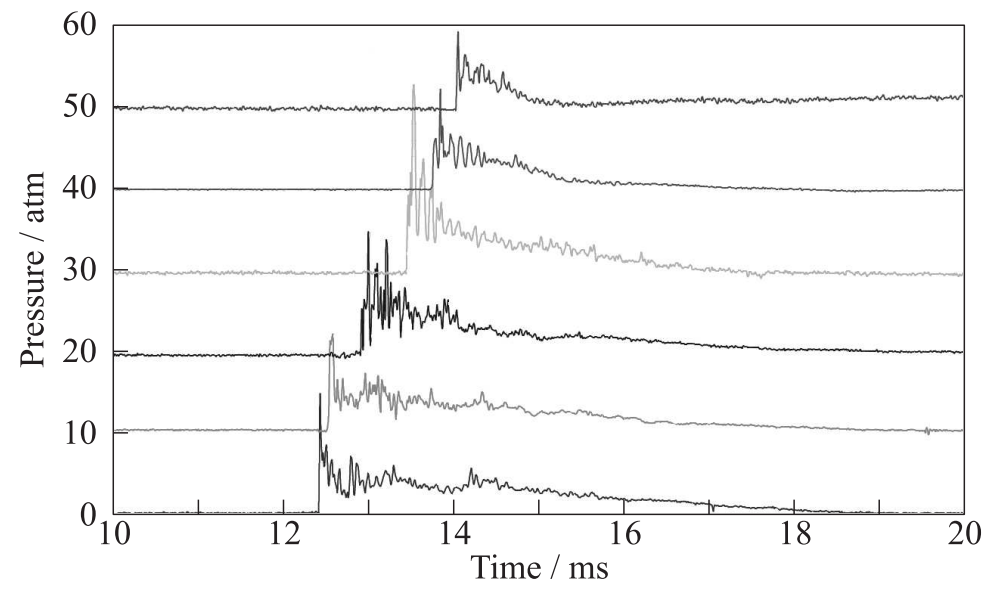

Figure 3 Pressure records in the tube with parabolic obstacles in the stoichiometric propylene-air mixture [16]. The initial shock wave velocity is $1070 \pm 30 \mathrm{~m} / \mathrm{s}$ 
Table 1 Comparison of measured shock wave velocities in the tube with regular parabolic and square obstacles $[16]$

\begin{tabular}{crc}
\hline Measuring segment, & \multicolumn{2}{c}{ Shock wave velocity, m/s } \\
\cline { 2 - 3 } mm & parabolic & orifice plates \\
\hline $670-795$ & $1070 \pm 30$ & $1070 \pm 30$ \\
$795-1195$ & $1061 \pm 30$ & $714 \pm 20$ \\
$1195-1705$ & $836 \pm 25$ & $637 \pm 20$ \\
$1705-2025$ & $1025 \pm 30$ & $1000 \pm 30$ \\
$2025-2355$ & $1590 \pm 50$ & $970 \pm 30$ \\
\hline
\end{tabular}

(LPC) with the obstructed section. The total length of the tube was $2.5 \mathrm{~m}$. The HPC was initially separated from the LPC by the bursting diaphragm. The obstructed section was $100 \times 100 \mathrm{~mm}$ square channel $1 \mathrm{~m}$ long with regular obstacles. This section reproduced exactly the shapes and arrangements of the obstacles of Figs. $1 a$ and $1 b$. The experiments were made with the stoichiometric propylene-air mixture at normal initial conditions. The shock waves were generated by filling the HPC (up to 8 bar) with the stoichiometric propylene-oxygen mixture and igniting it with a standard spark plug. The reactive mixtures were prepared by partial pressures in two mixers. To register the propagation velocities of shock waves and detonations, 6 high-frequency pressure transducers were installed along the tube. The data acquisition system comprised an analog-todigital converter and a PC. The measuring errors of shock wave velocity and pressure were estimated as $3 \%$ and $30 \%$, respectively. The pressure records of the DDT in the tube with parabolically-shaped regular obstacles are shown in Fig. 3. Table 1 shows the corresponding data for two experiments with shaped obstacles and orifice plates at identical initial conditions. Clearly, the use of shaped obstacles made it possible to promote fast DDT, whereas the use of orifice plates did not result in the detonation onset.

\section{TUBE COILS}

As shown in the previous section, the curved surfaces are capable of promoting fast DDT due to the onset of exothermic centers in the core flow behind the propagating shock wave. In view of it, fast DDT could be expected to arise more readily in curved rather than straight tubes. Thus, tube coils and bends should promote fast DDT. As a matter of fact, the experimental and computational studies described in this section demonstrate that fast DDT can be facilitated by placing a tube coil on the way of the propagating shock wave. 


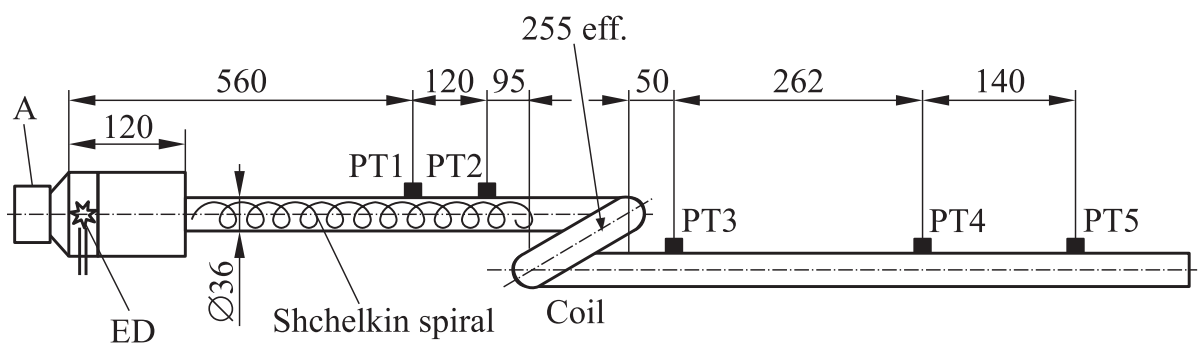

Figure 4 Schematic of the 36-millimeter inner diameter tube with a combination of Shchelkin spiral and tube coil $[19,20]$. Dimensions are in millimeters

Figure 4 shows the schematic of the tube $36 \mathrm{~mm}$ in diameter equipped with the air-assist atomizer A, electrical igniter ED, Shchelkin spiral and removable coil section with the coil length along the tube axis equal to $255 \mathrm{~mm}[19,20]$. A similar setup with a tube $28 \mathrm{~mm}$ in diameter was also used in the experiments. The air-assist atomizer provided full mass flow rate of the two-phase $n$-hexaneair mixture through the tubes. The mixture with the mean equivalence ratio of 1.3 was ignited by the three-electrode electrical igniter ED located $60 \mathrm{~mm}$ downstream from the atomizer nozzle where the mean drop size was 5-6 $\mu \mathrm{m}$. Shchelkin spiral $600 \mathrm{~mm}$ long wounded from steel wire $4 \mathrm{~mm}$ in diameter with a pitch of $18 \mathrm{~mm}$ was used for increasing turbulence intensity in the two-phase spray issuing from the atomizer nozzle. The new element - coil — was used for checking the possibility of fast DDT downstream from the spiral section. Highfrequency pressure transducers PT1-PT5 were used to register pressure histories and for determining pressure wave velocities.

In the experiments with the straight tube $36 \mathrm{~mm}$ in diameter with Shchelkin spiral but without coil, the shock wave velocity at the exit from the spiral attained $900-1000 \mathrm{~m} / \mathrm{s}$ and no fast DDT was detected. Variation of the spiral wire diameter as well as spiral pitch and length did not result in any significant changes in the resultant shock waves at ignition energies up to 240 J. Similar experiments were performed with the straight tube $28 \mathrm{~mm}$ in diameter with Shchelkin spiral. In this tube, fast DDT was not observed and the shock wave velocity registered at the exit from the spiral section was at the level of 800 $900 \mathrm{~m} / \mathrm{s}$.

Figure 5 shows the pressure histories detected by pressure transducers PT1PT5 in the 36-millimeter tube with Shchelkin spiral and coil at the ignition energy of $60 \mathrm{~J}$. In this experiment, fast DDT was detected. The shock wave velocity at the coil inlet was about $900 \mathrm{~m} / \mathrm{s}$. At the coil outlet (at a distance of about $1 \mathrm{~m}$ from the igniter), a detonation wave propagating at the velocity of $1750 \pm 20 \mathrm{~m} / \mathrm{s}$ was registered (see PT3 record). The detonation wave propagated toward the end of the tube with the constant mean velocity. Fast DDT is solely 


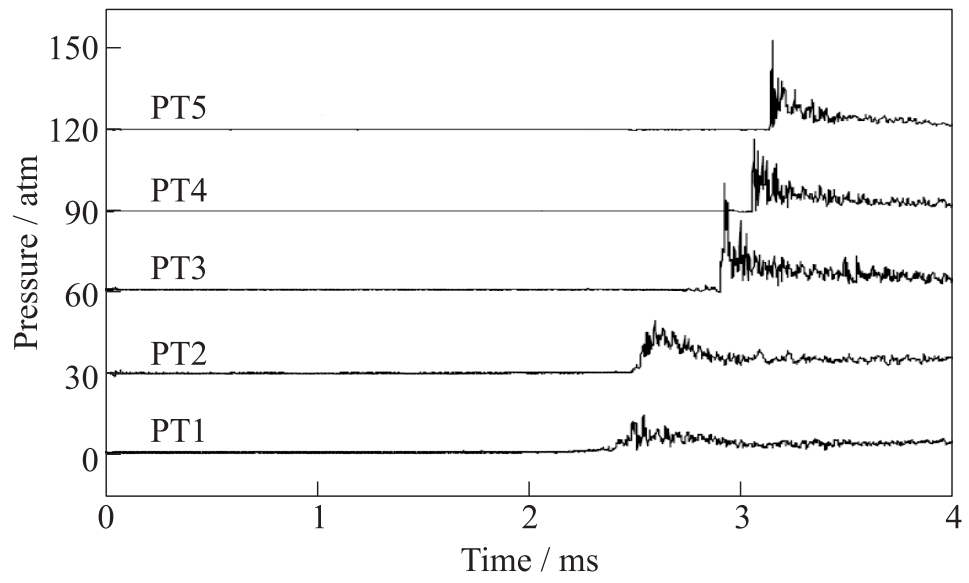

Figure 5 Pressure records in the run with low ignition energy [19, 20]

attributed to the use of the coil. Multiple reflections of the shock wave from the compressive surfaces inside the coil facilitate detonation initiation. Such shock reflections resemble those arising in the course of shock wave propagation in the straight channel with regular shaped obstacles.

In the computational studies, for resolving spatial effects inherent in shock wave propagation through a tube coil, the statement of the problem was based on three-dimensional (3D) compressible Euler equations coupled with the single-step kinetic equation for a single progress variable calibrated to represent the stoichiometric propane-air mixture. For solving the set of governing equations, a special parallel lower-upper symmetric Gauss-Seidel (LU-SGS) algorithm was developed based on the implicit finite-difference scheme applying finite-volume spatial discretization with first-order Harten-Lax-van Leer approximation of fluxes. The unstructured computational grids of tube coils contained up to 1,400,000 cells with the minimal and maximal cell sizes of 0.25 and $0.7 \mathrm{~mm}$, respectively. The time integration step was less than $1 \mu \mathrm{s}$. The calculations were performed using 32 processors MVS-15000BM of the Joint Supercomputer Center of the Russian Academy of Sciences and took in average 5 CPU hours per each run.

Shock-to-detonation transition in two configurations of tube coils filled with the stoichiometric propane-air mixture at normal conditions was considered. The first configuration was a circular 360-degree loop of the 28-millimeter tube $365 \mathrm{~mm}$ long (measured along the tube axis). The second configuration was a circular 360-degree loop of 36-millimeter tube $255 \mathrm{~mm}$ long (also measured along the tube axis). The inlet and outlet of each coil were positioned in the same normal plane and were in direct contact with each other. The primary shock wave at the coil inlet was modeled using Rankine-Hugoniot relationships at the shock discontinuity. 

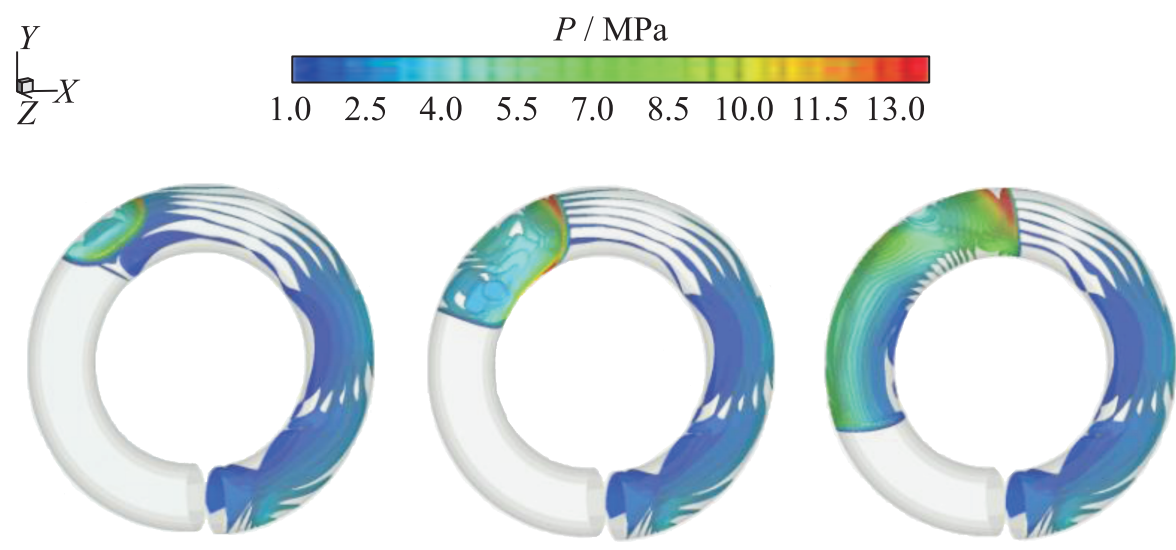

Figure 6 Evolution of pressure iso-surfaces in the 28-millimeter tube coil (first configuration) with the primary shock Mach number of 3.9. (Refer Frolov, p. 330.)

The results of calculations revealed salient features of fast DDT phenomena and were in qualitative agreement with the experimental observations. The minimal (critical) Mach numbers of the primary shock wave required for fast DDT in the coils of first and second configurations were 3.9 and 3.6, respectively. Several discrete locations of DDT were observed in the calculations in the vicinity to the collision sites of shock waves reflected from inner and outer tube walls. At near-critical conditions, fast DDT occurred in the second half-loop of the coil closer to the outlet (Fig. 6). With increasing the shock wave Mach number above the critical value, the transition region moved to the first half-loop of the coil closer to the inlet. The $3 \mathrm{D}$ effects were more pronounced for the tube coil of the second configuration with a smaller curvature radius and manifested themselves in the considerable deviation of the lead shock front from the planar shape. The optimal coil curvature is the issue of further studies.

\section{U-BENDS}

Tube bends are the elements which can be used to ensure compact design of multitube PDEs. Surprisingly little work has been done on the reactive shock and detonation diffraction in such elements [21]. Recent studies of DDT in curved tubes [22-25] have unequivocally demonstrated that tube curvature promoted fast DDT efficiently. The experimental and computational results were reported $[22,23]$ for fast DDT in the stoichiometric propane-air mixture in a tube with a single U-bend of the internal radius equal to tube diameter $(51 \mathrm{~mm})$. The results demonstrated a considerable effect of the U-bend on detonation ini- 


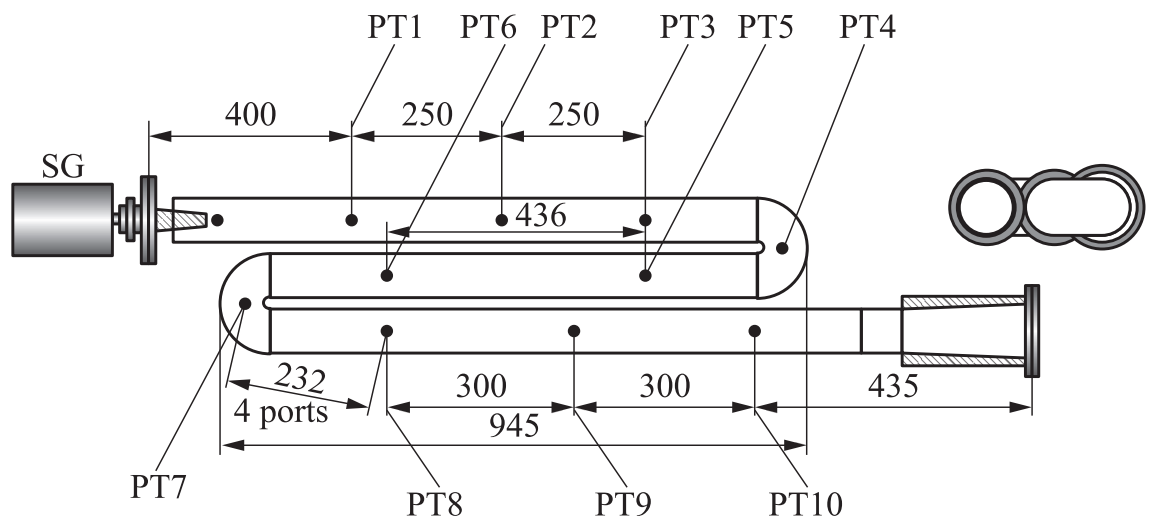

Figure 7 Schematic of the 51-millimeter tube with an SG and two U-bends. Dimensions are in millimeters. Dots indicate the positions of pressure transducers

tiation. The U-bend of the tube promoted fast DDT: a shock wave entering the U-bend at a velocity exceeding $1100 \mathrm{~m} / \mathrm{s}$ always transitioned to a detonation. The curvature of the U-bend, tube diameter, and compression phase duration of the initiating shock wave seem to be the most important governing parameters of the problem which determine the evolution of the initiating shock wave in such a system. In this section, some experimental and computational data on propagation of reactive shock waves in tubes with U-bends of the limiting curvature are discussed [24, 25].

The experimental setup is shown in Fig. 7. The setup comprised the detonation tube of round cross section with two U-bends. The tube was fixed at the experimental stand which was equipped with the utilities required for working with gaseous explosive mixtures. The explosive mixture was the stoichiometric propane-air. The mixture was prepared in the mixer at normal atmospheric conditions. At one end of the tube, a shock generator (SG) was mounted. Two types of SG were used: solid-propellant SG and electric-discharge SG [22-25].

The tube $51 \mathrm{~mm}$ in inner diameter had three straight sections and two Ubends, both in one plane. The internal radius of the U-bends was $11 \mathrm{~mm}$ while the axial radius was $37 \mathrm{~mm}$. Each U-bend was fabricated by welding four curved segments. The internal tube walls were smooth. Up to ten piezoelectric pressure transducers (PT1 to PT10) were mounted along the tube. The accuracy of shock wave velocity measurements was estimated as $4 \%$. The data acquisition system was triggered by pressure transducer PT1.

Figure 8 shows the variation of the mean shock wave velocity along the tube with the initial shock wave velocities in the range from 850 to $1300 \mathrm{~m} / \mathrm{s}$ at the first measuring segment. In this experimental series, the shock waves transitioned to detonations after passing either the first U-bend, or the second U-bend, i.e., fast 


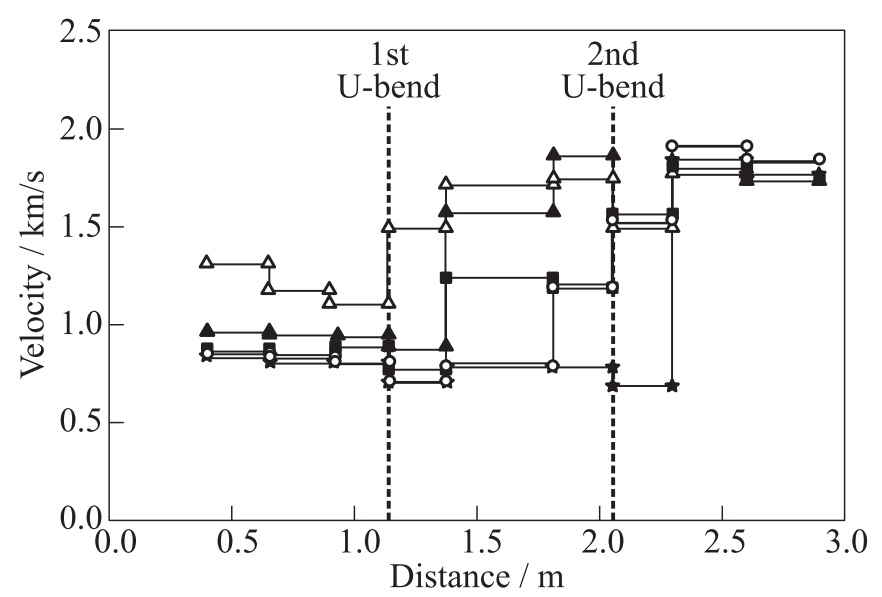

Figure 8 Mean shock wave velocities at different measuring segments of the tube with two U-bends (shown by vertical lines). Symbols are used to identify different runs

DDT phenomenon was detected. The lowest mean velocity of the primary shock wave entering the first U-bend and leading to the detonation onset behind the second U-bend was about $800 \mathrm{~m} / \mathrm{s}$. This velocity value should be treated as the critical condition for the setup of Fig. 7. Remind that in the experiments with a U-bend of smaller curvature [22, 23], the critical shock wave velocity was about $1100 \mathrm{~m} / \mathrm{s}$. It is seen from Fig. 8 that the higher the primary shock velocity the faster is the onset of detonation.

The mathematical model was based on the standard 2D Euler equations, energy conservation equation with a chemical source term, and equation of chemical kinetics for the stoichiometric propane-air mixtrure. The kinetics of propane oxidation was modeled either by a single-stage overall reaction or using a 5-step overall mechanism. A more detailed description of the numerical procedure is available elsewhere [22-25]. The computational studies revealed the important effect of the compression phase duration in the primary shock wave both on fast DDT and on the dynamic detonation structure. Figure 9 shows the situation when the primary shock wave transitions to a detonation behind the first U-bend. Interestingly, a slightly overdriven detonation arising behind the first U-bend suddenly decays behind the second U-bend due to the effect of a strong rarefaction wave and reignites as a single-cell CJ detonation. There are still a number of issues to be studied for such tube configurations, in particular, the effect of the distance between the bends on the evolution of the shock wave. The calculations (and experiments) show [23, 24] that stronger primary shock waves transition to a detonation behind the first U-bend, whereas weaker primary shock waves transition to a detonation only behind the second U-bend. 

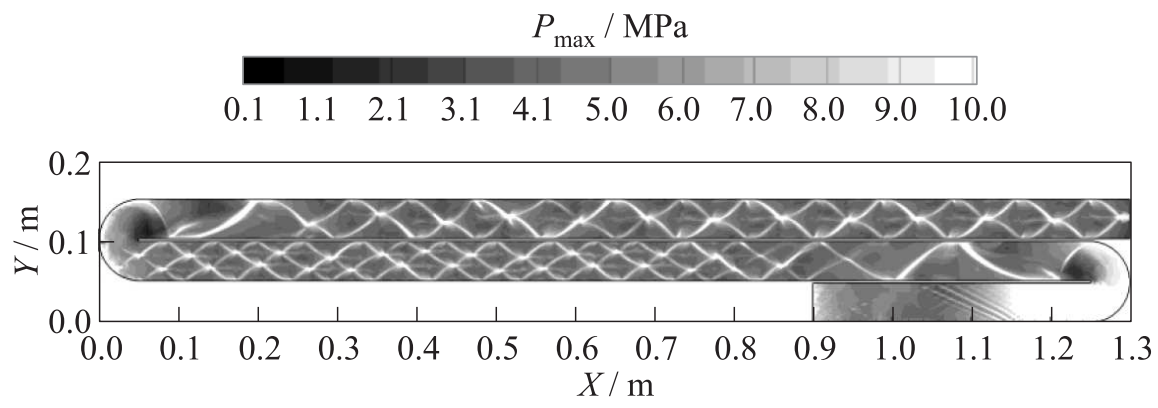

Figure 9 Maximum pressure records in the 51-millimeter channel with two U-bends of limiting curvature. The initial velocity and compression phase duration at the entrance to the first U-bend are $D=0.64 D_{0}$ and $\tau_{+}=600 \mu \mathrm{s}$

Nevertheless, depending on the distance between the U-bends, the detonation arising behind the first U-bend can decay behind the second U-bend. As a result, the use of weaker primary shock waves can surprisingly appear to be more beneficial for obtaining fast DDT than the use of stronger primary shock waves.

\section{DEFLAGRATION-TO-DETONATION TRANSITION IN LIQUID HYDROCARBON-AIR MIXTURES}

Low detonability of jet propulsion kerosene in air is the key barrier for the progress in the development of air-breathing PDE. In view of it, arranging of fast DDT seems to be a promising solution. As a matter of fact, the experimental research outlined in this section demonstrates that the use of fast DDT concept allows one to initiate a detonation of jet propulsion kerosene TS-1 (Russian analog of Jet-A) at short run-up distance and time applying a very small ignition energy [26].

Figure 10 shows the experimental setup, comprising injector 1, detonation tube 2 , igniter 3 , pressure transducers 4 , detonation arrester 5 , air bottle 6 , fuel valve 7 , air compressor 8 , kerosene tank 9, fuel filter 10, digital controller 11 , power supply 12 , PC 13 , control relay 14 , prevaporizer 15 , thermostat 16 , electrical heaters 17 and 18 , and thermocouples 19 . The fuel and air supply system provides the supply of fuel mixture components in constant proportion due to the same driving pressure. Mixing of fuel and air starts in the air-assist atomizer and terminates in the detonation tube of internal diameter $51 \mathrm{~mm}$ and $3 \mathrm{~m}$ long. The air-assist atomizer provides very fine kerosene drops mainly 5 to $10 \mu \mathrm{m}$ in diameter (measured by the phase Doppler particle analyzer (PDPA) and soot- 


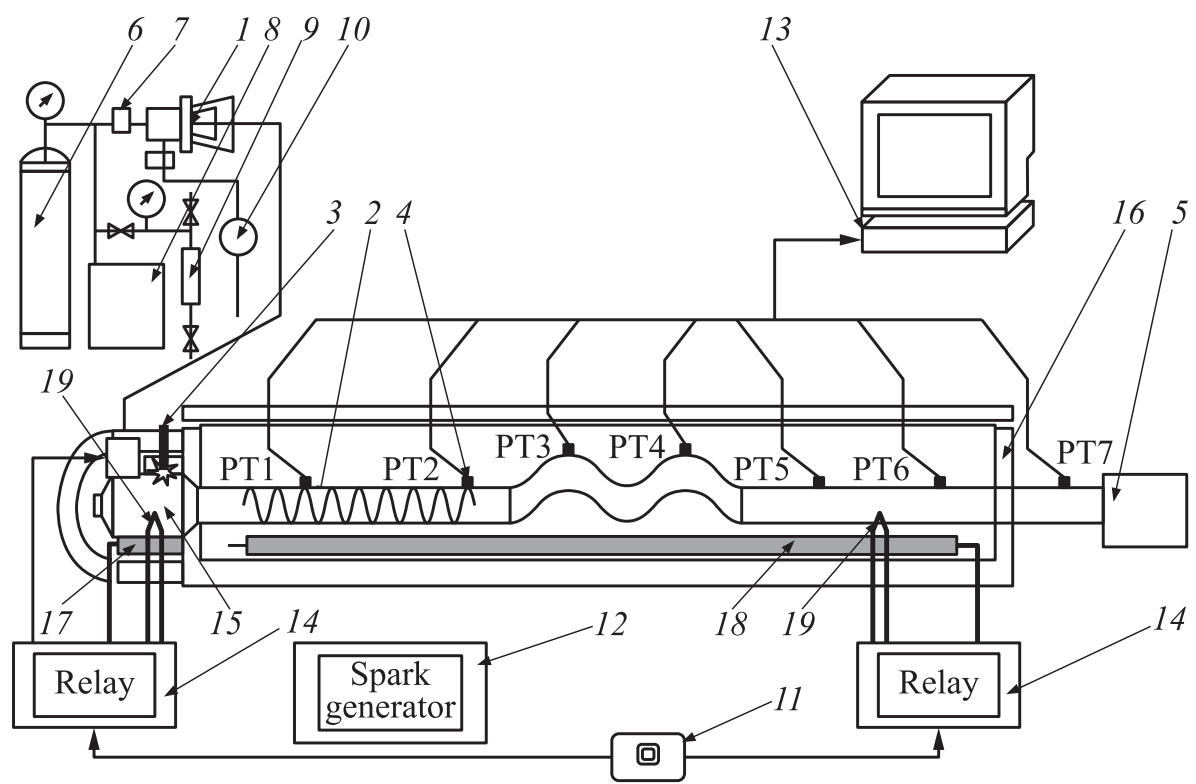

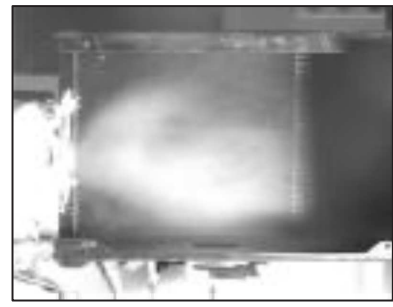

A

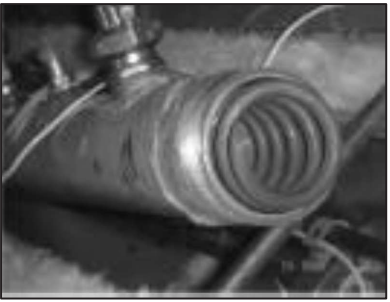

$\mathrm{B}$

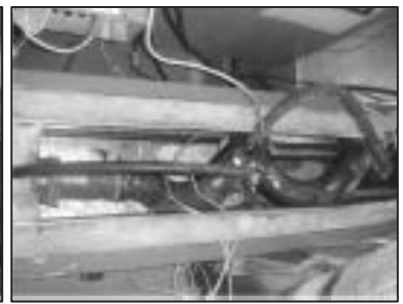

$\mathrm{C}$

Figure 10 Shchematic of the experimental setup with a kerosene prevaporizer (A), Shchelkin spiral (B), and 2-coil tube section enhancing fast DDT (C)

sampling method $[10,11])$. The two-phase fuel-air mixture of equivalence ratio $1.2 \pm 0.1$ is continuously injected to the prevaporizer section 15 of the detonation tube with the total mass flow rate at the level of $80 \mathrm{~g} / \mathrm{s}$. In this section, kerosene drops are partly vaporized and the hybrid drop-vapor-air mixture (insert A in Fig. 10) follows to the tube section with Shchelkin spiral (insert B in Fig. 10) and a removable 2-coil tube segment (insert C in Fig. 10).

Two sets of experiments have been made. In the first set, the detonation tube was straight, while in the second, it contained a 2-coil tube segment. The length of the Shchelkin spiral in both detonation tubes was $800 \mathrm{~mm}$. The spiral was mounted $70 \mathrm{~mm}$ downstream from the prevaporizer nozzle. In the experiments with both tubes, the prevaporizer wall temperature was $190 \pm 10^{\circ} \mathrm{C}$. 


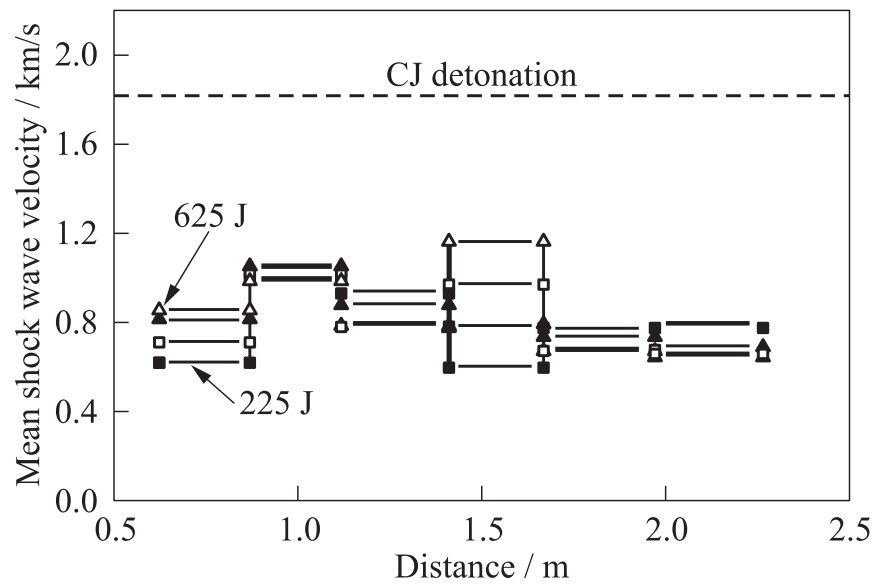

Figure 11 Measured mean shock wave velocities as a function of distance from the igniter in 4 runs with the ignition energy varied from 225 to $625 \mathrm{~J}$

The temperature of the tube segment with the Shchelkin spiral was $120-130{ }^{\circ} \mathrm{C}$ and the temperature of the tube segment up to pressure transducer PT6 was $110-120^{\circ} \mathrm{C}$. The temperature of the tube segment downstream from pressure transducer PT6 was $20-30{ }^{\circ} \mathrm{C}$. The (continuously) moving fuel-air mixture was ignited in the prevaporizer either by a standard spark plug or by the threeelectrode discharge [11]. The results of experiments were well reproducible once the ignition was triggered after the tube was purged by the reactive mixture in the amount of 3-4 tube volumes.

In the experiments with the straight tube, the ignition energy was varied from 5 to $700 \mathrm{~J}$. The maximum registered shock wave velocity in the straight tube was about $1200 \mathrm{~m} / \mathrm{s}$ (Fig. 11).

The second experimental series was performed with the 2-coil tube segment. This tube segment consisted of two complete turns of the tube with the external diameter of $57 \mathrm{~mm}$ tightly around a rod $28 \mathrm{~mm}$ in diameter with the pitch of $255 \mathrm{~mm}$. The curved tube segment was mounted $100 \mathrm{~mm}$ downstream from the end of the Shchelkin spiral. The ignition energy was varied from 5 to $176 \mathrm{~J}$. In these experiments, we have repeatedly registered detonation even at the lowest ignition energy used $(5 \mathrm{~J})$. Figure 12 shows the example of pressure records by pressure transducers PT1 to PT7 at the ignition energy of $5 \mathrm{~J}$ indicating fast DDT with the onset of detonation between PT5 and PT6. Figure 13 shows the measured mean shock wave velocities along the detonation tube in 12 runs with the ignition energy ranging from 5 to $100 \mathrm{~J}$. Clearly, fast DDT in kerosene-air mixture was repeatedly attained at a distance of about $2 \mathrm{~m}$ even at an ignition energy of $5 \mathrm{~J}$. This effect is solely attributed to the use of the curved tube segment. 


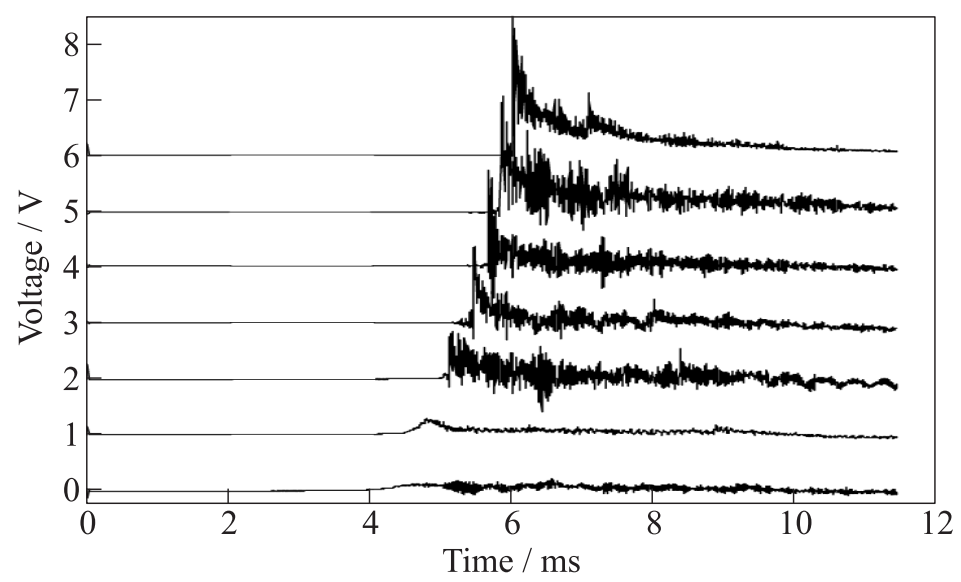

Figure 12 Pressure records in the run with igniter energy of $5 \mathrm{~J}$

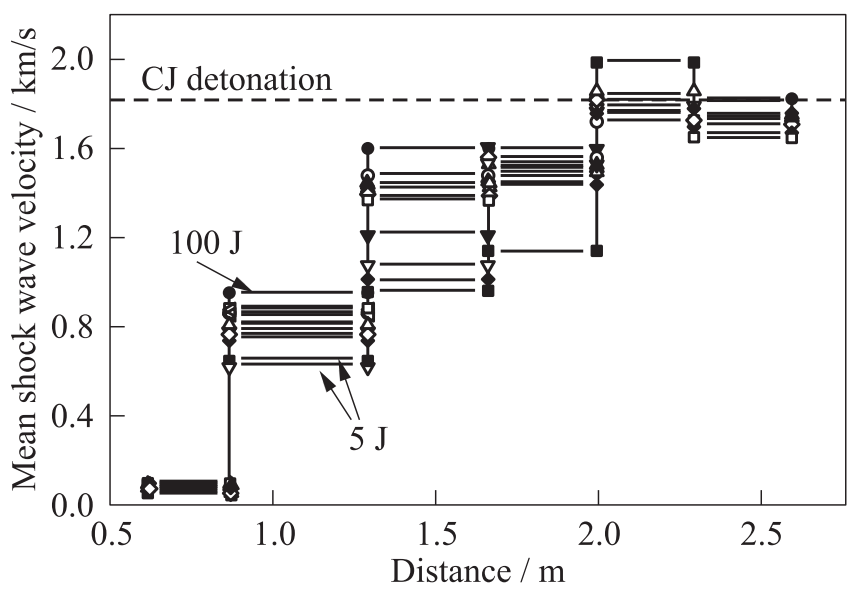

Figure 13 Measured mean shock wave velocities as a function of distance from the igniter in 12 runs with the ignition energy varied from 5 to $100 \mathrm{~J}$

To further decrease the ignition energy, a flame-jet igniter operating on the principle of a classical prechamber with a standard spark plug was designed and fabricated. The igniter operating either on a two-phase gasoline A80 (Octane number 80 ) - air or kerosene TS-1-air mixture was attached to the detonation tube of Fig. 10 instead of the prevaporizer. Before ignition, the tube was purged by the two-phase mixture issuing through the igniter nozzle. Spark ignition of the mixture in the igniter (ignition energy $100 \mathrm{~mJ}$ ) resulted in the energetic flame jet issuing through the igniter nozzle (Fig. 14). As fol- 


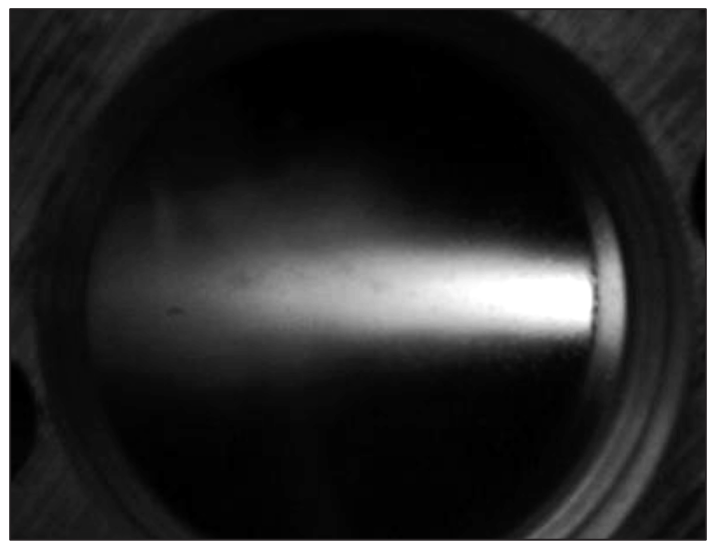

Figure 14 Flame jet issuing from the igniter nozzle at ignition energy of $100 \mathrm{~mJ}$

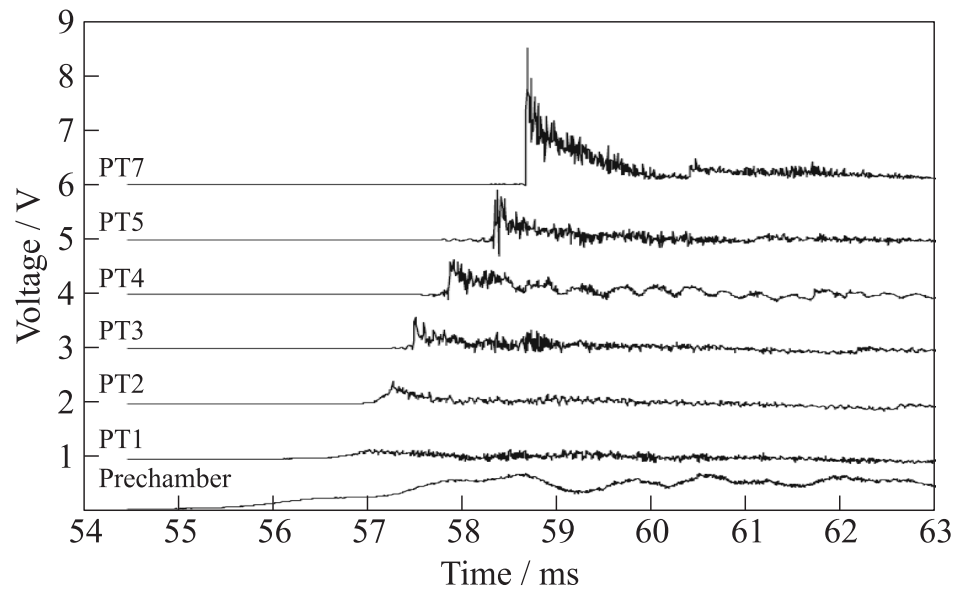

Figure 15 Detonation initiation by a flame-jet igniter in the gasoline A-80-air mixture

lows from Fig. 15, this flame jet is capable of initiating a detonation via fast DDT in the 2-coil tube segment. Clearly, the concept of flame-jet ignition offers a promising way of decreasing the detonation ignition energy down to fractions of joule. The total process time from mixture ignition in the igniter to detonation initiation was about $10.5 \mathrm{~ms}$. The DDT time after the flame jet entered the detonation tube was about 5-6 ms. In recent experiments with kerosene-air mixture, multipulse (up to 40) operation of the setup of Fig. 10 
with the frequency 2 to $10 \mathrm{~Hz}$ at ignition energy $100 \mathrm{~mJ}$ was successfully demonstrated.

\section{CONCLUDING REMARKS}

A concept of fast DDT [9] was applied to reduce the DDT run-up distance and time for air-breathing PDE applications. Various novel approaches were suggested and tested experimentally and computationally, namely, stimulation of fast DDT by using $(i)$ traveling ignition sources; $(i i)$ tube segments with regular shaped obstacles; and (iii) tube coils and U-bends. The use of certain combinations of these approaches made it possible to achieve fast DDT in kerosene-air mixture in a 51-millimeter tube with the run-up distance and time of about $2 \mathrm{~m}$ and 5-6 ms, respectively, applying spark ignition energy as low as $5 \mathrm{~J}$. The use of a flame-jet igniter allowed decreasing the detonation ignition energy down to $100 \mathrm{~mJ}$ for gasoline-air and kerosene-air mixtures while keeping the run-up distance and time at the same level.

Despite many promising features of the proposed approaches to reduce the DDT run-up distance and time for realistic PDE fuels, there are still many issues to address. One of the issues is that application of shaped obstacles, coils, Ubends, etc. results in the elevation of pressure loss which is crucial for propulsion. In view of it, one could expect that the DDT length and time reduction will require a sort of trade-off with other operational requirements for PDE-based propulsion.

\section{ACKNOWLEDGMENTS}

I would like to acknowledge valuable contribution of Akhmedyanov I. F., Aksenov V.S., Basevich V. Ya., Komissarov P. V., Markov V. V., Semenov I. V., Shamshin I. O., and Utkin A.S. to the research reviewed in this paper. The research was partly supported by the Russian Foundation for Basic Research, ONR, and ISTC.

\section{REFERENCES}

1. Bone, W. A., and R. P. Fraser. 1929. A photographic investigation of flame movements in carbonic oxide-oxygen explosions. Philos. Trans. R. Soc. London, Ser. A 228:197-234.

2. Zel'dovich, Ya. B. 1944. Theory of combustion and detonation of gases. Moscow: USSR Acad. Sci. Publ. 
3. Shchelkin, K. I. 1949. Fast combustion and spinning detonation of gases. Moscow: Voenizdat.

4. Oppenheim, A.K. 1972. Introduction to gasdynamics of explosions. Wien-NY: Springer.

5. Lee, J.H.S., and I. Moen. 1980. The mechans of transition from deflagration to detonation in vapor cloud explosions. Prog. Energy Combust. Sci. 6(4):359-89.

6. Higgins, A. J., P. Pinard, A. C. Yoshinaka, and J. H.S Lee. 2001. Sensitization of fuel-air mixtures for deflagration-to-detonation transition. In: High-speed deflagration and detonation: Fundamentals and control. Eds. G. Roy, S. Frolov, D. Netzer, and A. Borisov. Moscow: ELEX-KM Publ. 45-62.

7. Frolov, S. M., V. Y. Basevich, V.S. Aksenov, and S. A. Polikhov. 2003. Detonation initiation by controlled triggering of electric discharges. J. Propul. Power 19(4):57380.

8. Frolov, S. M., V. Ya. Basevich, V. S. Aksenov, and S. A. Polikhov. 2005. Spray detonation initiation by controlled triggering of electric dischargers. J. Propul. Power 21(1):54-64.

9. Frolov, S. M. 2006. Initiation of strong reactive shocks and detonation by traveling ignition pulses. J. Loss Prevention 19(2-3):238-44.

10. Frolov, S. M. 2006. Liquid-fueled, air-breathing pulse detonation engine demonstrator: Operation principles and performance. J. Propul. Power 22(6):1162-69.

11. Frolov, S. M., V. Ya. Basevich, V.S. Aksenov, and S. A. Polikhov. 2005. Optimization study of spray detonation initiation by electric discharges. Int. J. Shock Waves 14(3):175-86.

12. Basevich, V.Ya., S. M. Frolov, and V.S. Posvyanskii. 2005. Existence conditions for steady-state heterogeneous detonations. Rus. J. Chem. Phys. 24(7):58-68.

13. Peraldi, O., R. Knystautas, and J.H. S. Lee. 1986. Criteria for transition to detonation in tubes. Proc. Combust. Inst. 21:1629-37.

14. Basevich, V. Ya., V. I. Vedeneev, S. M. Frolov, and L. B. Romanovich. 2006. Nonextensive principle of constructing oxidation and combustion mechanisms for normal alkane hydrocarbons: Transition from $\mathrm{C}_{1}-\mathrm{C}_{2}$ to $\mathrm{C}_{3} \mathrm{H}_{8}$. Rus. J. Chem. Phys. 25:8796.

15. Semenov, I., S. Frolov, V. Markov, and P. Utkin. 2006. Shock-to-detonation transition in tubes with shaped obstacles. In: Pulsed and continuous detonations. Eds. G. Roy, S. Frolov, and J. Sinibaldi. Moscow: TORUS PRESS. 159-69.

16. Frolov, S. M., I. V. Semenov, P. V. Komissarov, et al. 2007. Reduction of the deflagration-to-detonation transition distance and time in a tube with regular shaped obstacles. Doklady Phys. Chem. 415(Part 2):209-13.

17. Sturtervant, B., and V.A. Kulkarny. 1976. The focusing of weak shock waves. J. Fluid Mech. 73:651-71.

18. Zel'dovich, Y.B., B.E. Gelfand, S. A. Tsyganov, S. M. Frolov, and A. N. Polenov. 1988. Concentration and temperature nonuniformities of combustible mixtures as reason for pressure waves generation. In: Dynamics of explosions. Progress in astronautics and aeronautics ser. Eds. A.L. Kuhl, J. R. Bowen, J.-C. Leyer, and A. A. Borisov. 114:99-123. 
19. Frolov, S. M., V.S. Aksenov, and V. Ya. Basevich. 2005. Decreasing the predetonation distance in a drop explosive mixture by combined means. Doklady Phys. Chem. 401(1):28-31.

20. Frolov, S. M., V.S. Aksenov, and V. Ya. Basevich. 2006. Initiation of heterogeneous detonation in tubes with coils and Shchelkin spiral. High Temp. 44(2):283-90.

21. Nettleton, M. A. 1987. Gaseous detonations. London-NY: Chapman and Hall.

22. Frolov, S. M., V.S. Aksenov, and I. O. Shamshin. 2005. Detonation propagation through U-bends. In: Nonequilibrium processes. Vol. 1. Combustion and detonation. Eds. G. D. Roy, S. M. Frolov, and A. M. Starik. Moscow: TORUS PRESS. 348-64.

23. Frolov, S. M., V. S. Aksenov, and I. O. Shamshin. 2007. Shock wave and detonation propagation through U-bend tubes. Proc. Combustion Institute 31:2421-28.

24. Frolov, S. M., V.S. Aksenov, and I. O. Shamshin. 2007. Reactive shock and detonation propagation in U-bend tubes. J. Loss Prevention 20(4-6):501-8.

25. Frolov, S. M., V.S. Aksenov, and I. O. Shamshin. 2007. Initiation of gaseous detonation in tubes with sharp U-bends. Doklady Phys. Chem. 417(Part 2):22-25.

26. Frolov, S. M., and V.S. Aksenov. 2007. Deflagration-to-detonation transition in a kerosene-air mixture. Doklady Phys. Chem. 416(Part 1):261-64. 